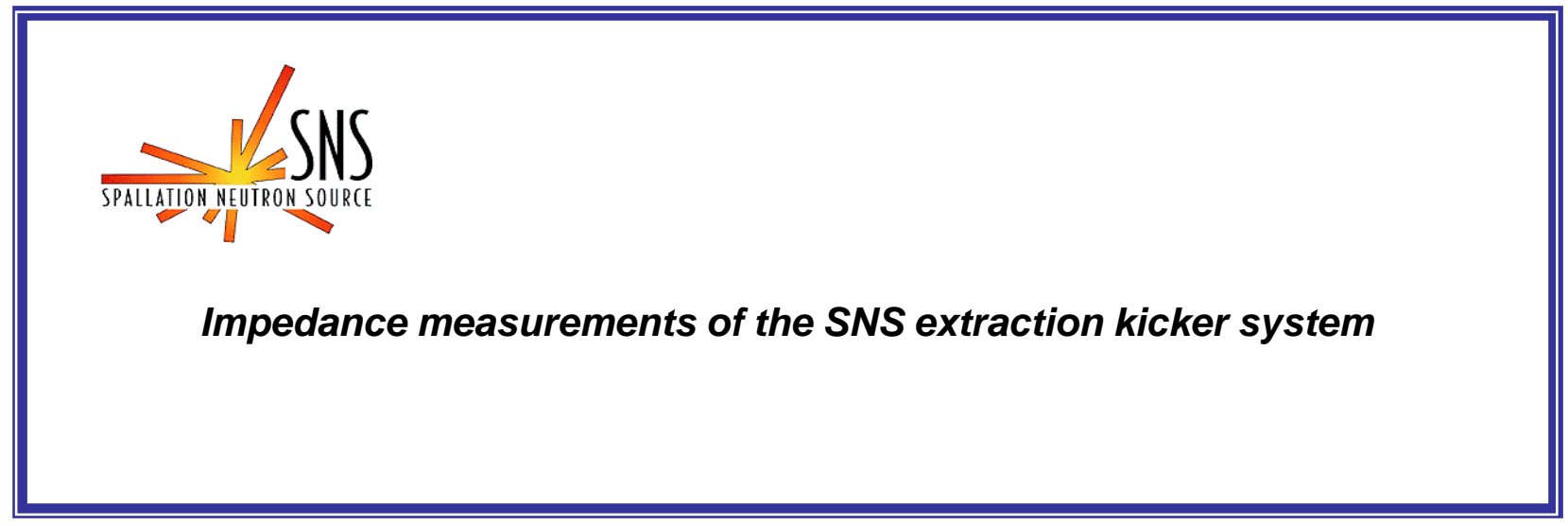

BNL/SNS TECHNICAL NOTE

NO. 135

H. Hahn

May 10, 2004

COLLIDER-ACCELERATOR DEPARTMENT BROOKHAVEN NATIONAL LABORATORY

UPTON, NEW YORK 11973 


\title{
Impedance measurements of the SNS extraction kicker system
}

\author{
H. Hahn
}

\section{Introduction}

The driving terms of instabilities in accelerator/storage rings depend on the beam surroundings which are conveniently described by impedances. Establishing and maintaining a coupling impedance budget becomes an important part of designing a high current accelerator. Theoretical estimates for typical accelerator components have been developed and are available in the standard literature. For critical devices, the estimates need to be confirmed by impedance bench measurements. The basic concept of bench measurements relies on simulating the beam by a wire for longitudinal or a twin wire Lecher line for transverse measurements. The measurements typically involve a measurement of the Device Under Test (DUT) and of a Reference structure with the difference or ratio of the data used to interpret the coupling impedance [1].

The theoretical basis and a method of transverse impedance measurements involving a single loop was reported in the seminal paper by Nassibian and Sacherer (NS) [2]. The by now standard method was first applied by Walling et al.[3] and involves the insertion of twin-wires into the magnet aperture, respectively the reference tube, and the measurement of the forward transmission scattering coefficients with a network analyzer. The various formulas for the interpretation of the data have been discussed at length in a recent paper [4]. Applying the wire method to frequencies at $\sim 1 \mathrm{MHz}$ and below entails difficulties due to noise and drift of the instrument, and other methods must be considered. For example, the transverse impedance study of the LHC dump kicker was performed by Mostacci et al. with multi-turn loops [5].

In the case of the Spallation Neutron Source (SNS) accumulator ring [6], the extraction kicker represents the most critical impedance item and the prototype magnet was intensively studied using the twin-wire method $[7,8,9,10]$. The immediate impetus for the present study came from the requirement of an improved impedance match of the kicker system involving the pulse forming network (PFN) and the long cable connections to the kicker magnet. The frequency range of interest to SNS covers frequencies, from $\sim 100 \mathrm{MHz}$ down to below $1 \mathrm{MHz}$. Especially at the very-low frequency end, the signals become very weak and the results appear extremely noisy and may no longer be valid. This provided the motivation to extend the study and to search for measurement methods beyond the obvious averaging and smoothing of the data.

The low-frequency range is on one hand the cause of the problem, but on the other hand it also presents the possibility of treating the magnet as lumped element transformer. It was already pointed out by Nassibian and Sacherer, that the coupling impedance is generated by the flux coupled to the external system plus a contribution from image currents. At low frequencies, the impedance is dominated by the coupled flux impedance which can be described by the transformer model. The model consists of the magnet inductance, with the external impedance in parallel across the bus-bar terminals. The 
mechanical construction of the SNS extraction kicker bus-bar provides a short gap, where a direct measurement of the coupled impedance can be done. A shorted bus-bar gap can also serve as reference for the direct measurement of the impedance of the magnet model components. The justification of the direct method and its limits are a paramount topic of the present paper [11].

Although a substantial data base for the SNS kicker was available from the previous measurements, the results in this paper represent new measurements, primarily addressing the PFN generated impedance. The extension to the low frequency end made it necessary to obtain continuously measured data over the entire frequency range of interest. In this paper, three different approaches, that is the twin wire measurement with external reference, the measurement with shorted busbar as reference, and the direct impedance measurements at the busbar are described and compared. Although dedicated to a specific kicker magnet, the discussion of the alternate methods is expected to be of more general interest.

\section{Twin-wire measurements}

The transverse coupling impedance of a kicker magnet can be measured on the bench by using the standard method in which a twin-wire (TW) "Lecher" line, simulating the beam, is inserted into the "Device Under Test (DUT)". Using a network analyzer, the forward transmission coefficients $S_{21}^{D U T}$ of the kicker is determined and compared with the $S_{21}^{R E F}$ obtained in a reference measurement. The theoretical reference for the measurement of the transverse coupling impedance is the free space. However, a "true" free space without radiation effects is difficult to arrange, and the use of a beam tube with the accelerator dimensions is considered as mandatory.

The scattering coefficients were measured with the network analyzer, Agilent 8753ES, set for a linear frequency range from $30 \mathrm{kHz}$ to $10 \mathrm{MHz}$, respectively to $100 \mathrm{MHz}$, with 1601 points. The preeminent, low-frequency instrument errors of noise and drift were reduced by using a $100 \mathrm{~Hz}$ IF-bandwidth and by limiting the time between measuring the DUT and the reference to minutes. The data of a magnet locally impedance-terminated can be corrected by smoothing, however the critical data of the "open" or the PFN terminated magnet with its sharp resonances should be obtained by averaging several sweeps.

The setup for a twin wire measurement of a lumped kicker magnet, such as the SNS extraction kicker, is symbolically represented by the equivalent circuit in Fig. 1. The magnet has an inductance, $L_{K}$, the TW line has an inductance, $L_{C}$ and a characteristic impedance, $R_{C}$. The coupling of the magnet to the TW line is given by the coupling coefficient, $\kappa$. As already discussed by NS, the total impedance of a lumped kicker magnet is composed of the coupled part, which is due to the external termination, $Z_{g}$ plus a uncoupled part due to image currents, represented by, $L_{I}$. The image current is primarily inductive but at higher frequencies can have a resistive part due to the ferrite 
material. The "stray" inductance, $\left(1-\kappa^{2}\right) L_{C}$, leads to a difference between the impedance seen by the beam and measured by the TW method. In a typical case with the wire spacing small compared to the magnet aperture, this difference is inductive and can be neglected in the instability driving resistive term. In this vein, small stray inductance values are not shown in the circuit, length differences between magnet and TW line are ignored, as well as a small difference in inductance and impedance between the line in the reference tube or in the magnet have been neglected. It is to be understood that the symbol values are approximate and may have to be adjusted and used with different notation for a specific measurement.

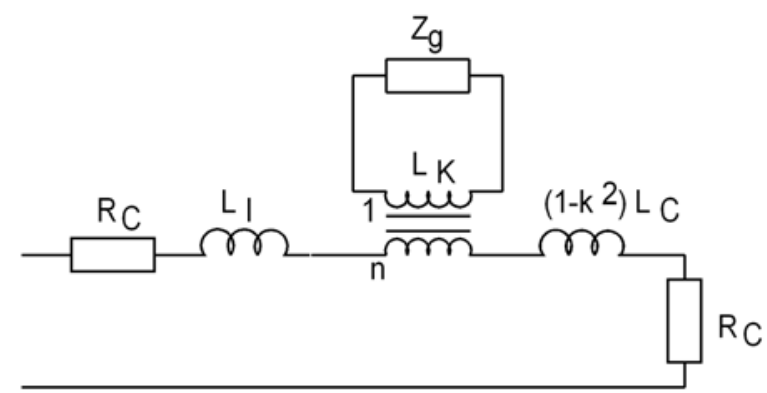

FIG. 1. Lumped kicker magnet circuit model

The equivalent circuit suggests an alternate reference set-up for the measurement of the coupled impedance part by shorting the magnet at the busbar gap. Errors due to mechanical changes or drift in the network analyzer are thus eliminated or at least reduced. The result for the termination generated resistive impedance component, which is of particular interest for the beam stability, remains unchanged, but the reactive part may differ from the tube reference result. Note that the image current and stray impedance is now part of the reference and is subtracted from the total impedance by this measurement. Furthermore, in the SNS kicker, the magnet geometry prevents a perfect short and the data requires a small correction and will be addressed in the sequel.

The scattering coefficients are interpreted according to the standard HP-formula for lumped elements [4],

$$
Z^{D U T}=2 R_{C}\left(S_{21}^{R E F} / S_{21}^{D U T}-1\right),
$$

with $R_{C}$ the nominal impedance to which the TW line is matched, not necessarily the actual, line impedance. Primary matching to the instrument impedance, $R_{0}=50 \Omega$ is done with transformers and completed with resistive matching. The characteristic line impedance is only approximately known, but mismatch errors are minimized and vanish at the low frequency end by the use of the nominal $R_{C}$. The transverse coupling impedance now follows from the standard twin-wire measurement expression, as [2]

$$
Z_{\perp}=\frac{c}{\omega \Delta^{2}} Z^{D U T}
$$

with $\Delta$ the effective wire spacing to be discussed below. The transverse impedance has values in vertical and horizontal direction. The measurements in vertical, that is the kick direction, are here identified by $Z_{y}$ rather than the generic $Z_{\perp}$. 
For the typical case in kicker magnets, one has $Z^{D U T} \ll R_{C}$, which leads to the alternative approximation for the transverse coupling impedance of the DUT, known as the log-formula [3],

$$
Z_{\perp}=\frac{c Z^{D U T}}{\omega \Delta^{2}} \approx 2 \frac{c R_{C}}{\omega \Delta^{2}} \ln \frac{S_{21}^{R E F}}{S_{21}^{D U T}}
$$

Unless noted differently, the results presented in this paper were obtained by using the conversion in the network analyzer corresponding to the standard lumped formula.

The accuracy of the resistive transverse coupling impedance is inherently noise limited at the low frequency end by the $\omega^{-1}$ factor. The reactive part of the DUT impedance frequently is proportional to $\omega$ and thus is less sensitive to noise. The irreducible noise in the $S_{21}$ measurement is $\sim 10^{-4}$ peak corresponding to $>1 \mathrm{k} \Omega / \mathrm{m}$ at $1 \mathrm{MHz}$ with the TW line used here, clearly preventing low-frequency measurements.

\section{Twin-Wire Lecher line}

The impedance measurements were performed by using a twin-wire line, $126 \mathrm{~cm}$ long, home-made from $5 \times 7.5 \mathrm{~mm}$ rectangular tubes as shown in Fig. 2. The wire spacing is adjustable allowing changes of the inductance, characteristic impedance, and the signal level. Clearly, the signal increases with the wire spacing and the signal to noise ratio is reduced. It is recommended to keep the wire spacing smaller than the half height of the magnet aperture in order to remain in the perturbation regime, here $d \ll 12.4 \mathrm{~cm}$.

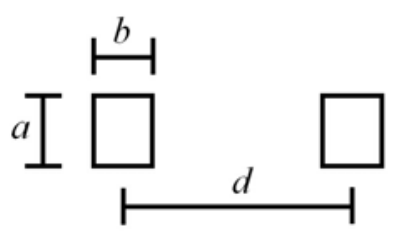

FIG. 2: The homemade twin-wire (TW) line. The dimensions are: $a=7.5 \mathrm{~mm}, b=5$ $\mathrm{mm}$, and $d=40.6 \mathrm{~mm}$.

\section{Effective wire spacing}

The transverse impedance value depends quadratically on the effective wire spacing, $\Delta$. Applying the theoretical results for round to rectangular wires, one finds the effective spacing to be

$$
\Delta=d \sqrt{1-(b / d)^{2}} \text {. }
$$

In the typical case of the wire size much smaller than the wire spacing, the effective $\Delta$ is given by the center-to-center distance of the wires. This was checked by measuring the peak impedance of the "open", i.e. un-terminated magnet, employing different TW-lines. Scaling of the peaks gave results which are consistent with the use of the simpler $\Delta \approx d$. For all measurements presented here an effective spacing of $\Delta=40.6 \mathrm{~mm}$ will be used. 


\section{Characteristic line impedance}

Interpretation of the measurements requires the knowledge of the characteristic impedance of the TW-line. The characteristic impedance was measured directly with the communication signal analyzer, Tektronix CSA 803 to be $Z_{L}=260 \pm 10 \Omega$.

The characteristic impedance has been estimated to be $Z_{L} \approx 277 \Omega$ from the theoretical expression for round wires by accommodating the rectangular lines with the use of $b^{\prime} \approx 2(a+b) / \pi$ instead of $\mathrm{b}$ in [12]

$$
Z_{L}=\frac{c \mu_{0}}{\pi} \operatorname{acosh}\left(d / b^{\prime}\right)
$$

An alternate determination of the characteristic impedance comes from the measurements of the line inductance per unit length. The TEM waves propagate on the TW line in air with the velocity of light, which implies the relation between characteristic impedance and inductance per unit length

$$
Z_{L}=c L_{L}^{\prime}
$$

The input impedance of the shorted TW-line was measured in air at $1.5915 \mathrm{MHz}$ corresponding to the inductance of $L_{L}^{\prime} \approx 0.96 \mu \mathrm{H} / \mathrm{m}$, a value closer to $Z_{L} \approx 288 \Omega$.

\section{Impedance Matching of the TW-line}

Although the bare TW-line can be used without matching at the low frequency end, $<10$ $\mathrm{MHz}$, to determine the basic kicker magnet properties, it is for most coupling impedance measurements necessary to match the characteristic impedance to the $50 \Omega$ instrument impedance value. This can be done by resistive impedance matching or by the use of a transformer, or by a combination thereof. Commercial (North Hills) wide-band transformers with a center-tapped secondary winding, serving as $180^{\circ}$ hybrid, were used for the measurements. Several transformers with different secondary impedance value and bandwidth were available and are identified according to their impedance as

$$
\begin{aligned}
& \text { X300 (NH15880 with } 100 \mathrm{kHz}-100 \mathrm{MHz} \text { ), } \\
& \text { X200-100 (NH15887 with } 100 \mathrm{kHz}-100 \mathrm{MHz} \text { ), } \\
& \text { X200-60 (NH15888 with } 10 \mathrm{kHz}-60 \mathrm{MHz} \text { ). }
\end{aligned}
$$

Matching is confirmed by measuring the input impedance of the input transformer when the output transformer is terminated with the instrument impedance. The objective is a frequency-independent input impedance. The input impedance of the twin line placed between the X300 and X200-100 transformers is shown in Fig. 3.
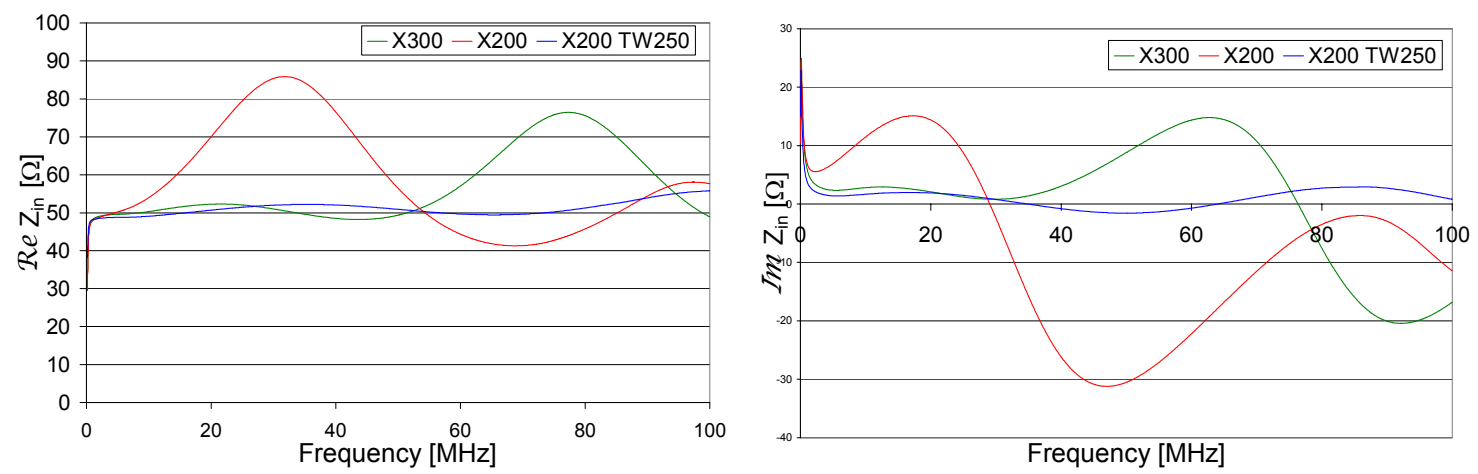

FIG. 3. Input impedance of twin line with different transformers 
A flat response (blue curve) was achieved by matching the line with the X200 transformer, $R_{0}=200 \Omega$ to $R_{C} \approx 250 \Omega$, the value to be used in the interpretative HPformula. Resistive matching is done on the input side with a parallel resistor, $R_{P}$, and a series resistor, $R_{i n}$,

$$
\begin{aligned}
& R_{P}=R_{0} \sqrt{R_{C} /\left(R_{C}-R_{0}\right)} \approx 447 \Omega, \\
& R_{\text {in }}=R_{C}-R_{0} R_{P} /\left(R_{0}+R_{P}\right)=2 \times 56 \Omega,
\end{aligned}
$$

and a series resistor on the output side, $R_{\text {out }}=R_{C}-R_{0}=2 \times 27 \Omega$.

The effect of the transformer bandwidth on the input impedance is shown in Fig. 4. Clearly, measurements below $\sim 60 \mathrm{MHz}$ are better done with the X200-60 transformers.
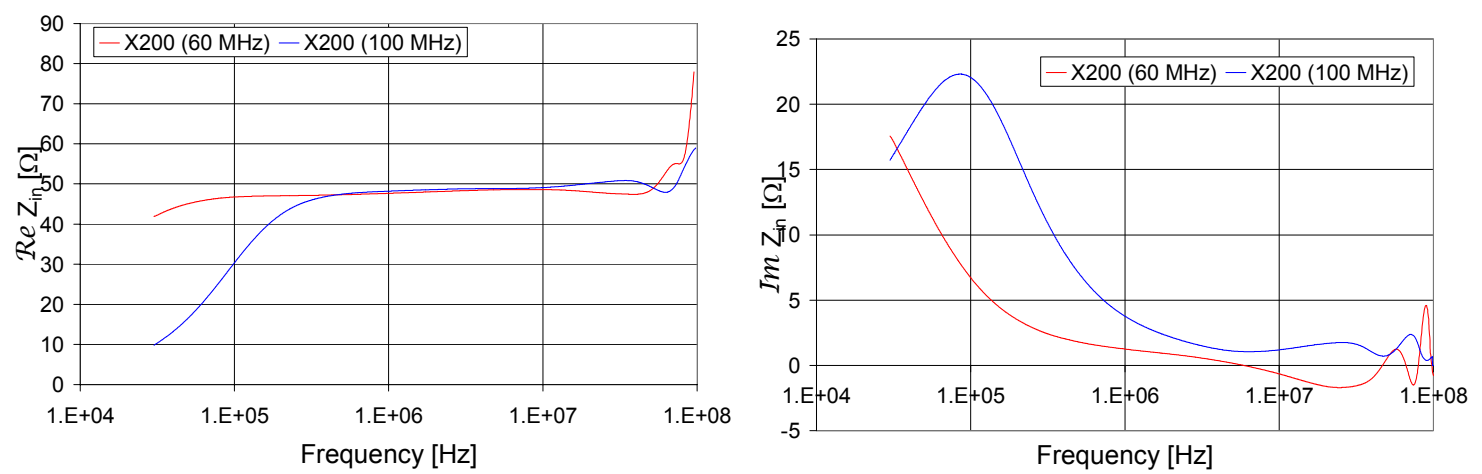

FIG. 4. Input impedance at transformers with different frequency range

\section{Reference tube calibration}

The TW line with transformer assembly provides the $S_{21}^{R E F}$ from a measurement in the reference tube. The forward scattering coefficient of the assembly with either X200 transformer, matched to $250 \Omega$, was measured in the tube and is shown in Fig. 5. The result is in reasonable agreement with the nominal value, $S_{21} \approx 0.55$. Measurements below $\sim 10 \mathrm{MHz}$ benefit from using the $60 \mathrm{MHz}$ transformer version, whereas the 100 $\mathrm{MHz}$ version is better suited to the upper frequency range. However, since the SNS stability considerations focus on $\sim 4 \mathrm{MHz}$ and $\sim 50 \mathrm{MHz}$, both transformers have been used for system measurements.
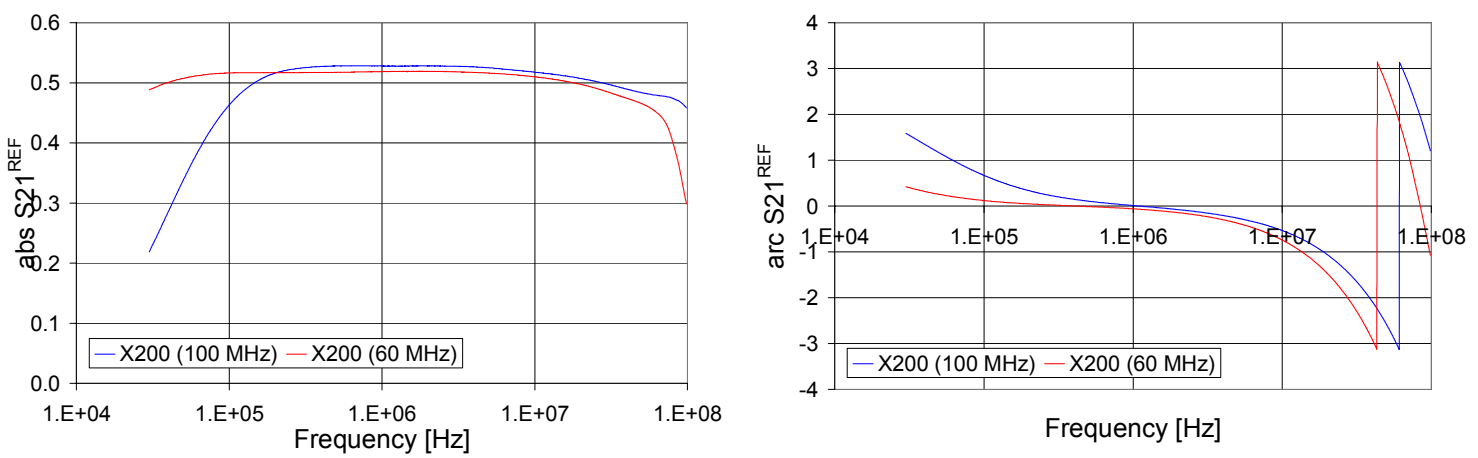

FIG. 5. Amplitude and phase of scattering coefficient in reference tube. 
Theoretical estimates of the transverse coupling impedance calculate the difference due to the ring component versus the beam in free space. The calibration of the TW line in a tube eliminates extraneous errors and differs from that in air only by the shielding effect, which appears as a reduction of the line inductance (and characteristic impedance) given by [5]

$$
\Delta L \approx \frac{2 \mu_{0} \ell}{\pi}\left(\frac{\Delta}{D}\right)^{2} .
$$

For the reference tube with diameter $D=0.150 \mathrm{~m}$ and length $\ell=0.94 \mathrm{~m}$, one finds $\Delta L=$ $0.05 \mu \mathrm{H}$, or scaled to the shorter magnet length , $\sim 0.02 \mu \mathrm{H}$, indeed comparable to random measuring errors. The measured impedance of the beam tube versus free space, shown in Fig. 6, was measured via the scattering coefficients and, in view of its length, was interpreted with the log formula

$$
Z^{D U T}=-2 R_{C} \ln \frac{S_{21}^{T U B E}}{S_{21}^{A L R}}
$$
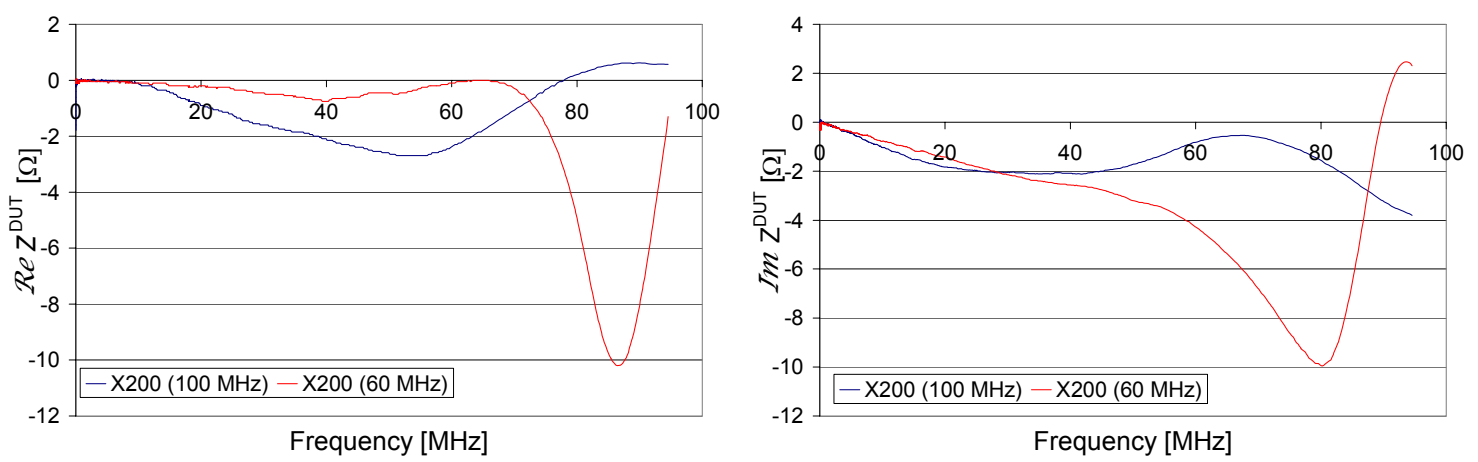

FIG. 6 Beam tube impedance versus free space.

The impedance of the beam tube is due to the resistive wall impedance plus the screening effect, both of which are expected to be monotonic with increasing frequency. The results in Fig. 6 are not monotonic with frequency and pose interpretation problems. At low frequencies, below $\sim 10 \mathrm{MHz}$, both transformers give the same resistive impedance and show the inductance change of $\sim 0.013 \mu \mathrm{H}$, although significantly lower than the theoretical estimate. The origin of the difference in the resistive component at higher frequencies is believed to be mostly transformer induced although some radiation effects were also observed. Although the coupling impedance measurement is obtained from the $S_{21}$ ratio to eliminate the line properties, the difference in results point to the possibility of significant errors at frequencies above $\sim 50 \mathrm{MHz}$, considering the comparable size of the kicker magnet and apparent beam tube impedance. For example, the SNS extraction magnet with the $25 \Omega$ PFN termination shows a scaled impedance of $25 \times(4.06 / 24.8)^{2} \approx 0.7 \Omega$, which is smaller than the apparent beam tube value.

In view of these difficulties, attempts to use free space for the reference calibration was abandoned. It also followed that the low and high frequency range is best covered with the $60 \mathrm{MHz}$ or $100 \mathrm{MHz}$ transformer respectively. Furthermore, coupling impedance results including the reactive component must be obtained with the beam tube 
calibration. As demonstrated below, reliable low frequency results for the resistive impedance part can also be obtained by using the shorted bus-bar as reference, or from the direct bus-bar input impedance measurement.

\section{The SNS Extraction Kicker Magnet Prototype}

A full-size prototype of the SNS extraction kicker magnet served for the studies reported here. A schematic view of the free standing window frame magnet as well as the magnet installed in its vacuum vessel is shown in Fig. 7. The vessel picture shows the busbar, its connection to the outside via the feedthrough, and the straps at the side connecting the upper and lower busbar plates. The busbar and ferrites are fully isolated from the vessel by ceramic plates. So-called eddy current strips to reduce longitudinal impedance are also visible. The ferrite type is CMD5005 (Ceramic Magnetics Inc., Fairfield, NJ, USA).

The kicker deflects vertically and has the height, width and length dimensions of $h=$ $24.8 \mathrm{~cm}, w=15.9 \mathrm{~cm}, l=36 \mathrm{~cm}$ and the ferrite thickness $t=2.54 \mathrm{~cm}$. The busbar is 40 $\mathrm{cm}$ long, and the vacuum vessel has a $62 \mathrm{~cm}$ inner diameter and it is $88 \mathrm{~cm}$ long. Note the busbar end plates which represent a non-negligible capacity in the unconnected ("bare") magnet.
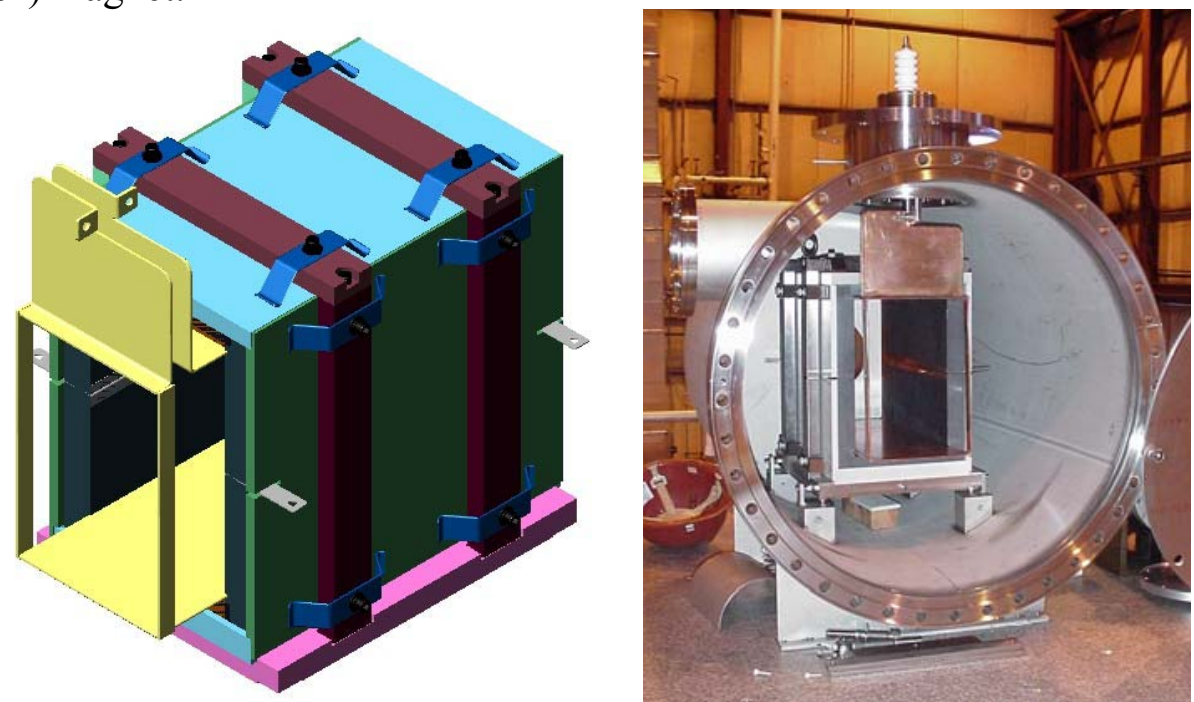

FIG.7. The SNS extraction kicker: schematic view (left), the kicker in the vessel (right).

The electrical properties of the bare magnet are obtained from input impedance measurements at the busbar, using the $S_{11}$ function of the network analyzer. The instrument cable is connected via clip-leads, which alone represent an inductance of $0.061 \mu \mathrm{H}$ and a capacity of $5.7 \mathrm{pF}$ which, since it is not part of the instrument calibration, needs to be subtracted from the measured data. A copper strap with clip-leads used as busbar short has an inductance of $\sim 0.06 \mu \mathrm{H}$, and must be considered when the short is taken as reference. The real and imaginary input impedance at the busbar is shown in Fig. 8 for the two cases of open and $25 \Omega$ termination. 

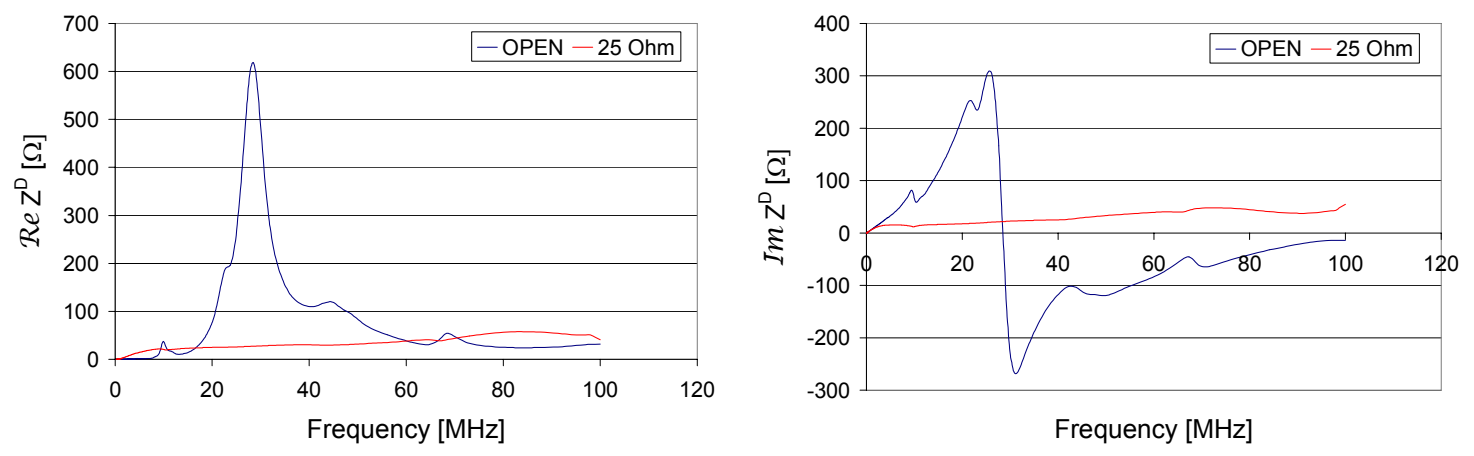

FIG. 8. Input impedance of the prototype kicker magnet at the busbar

The input impedance measurement yields accurate results at the low frequency range from $\sim 1$ to $10 \mathrm{MHz}$ which is of particular interest to the SNS stability considerations. The bare magnet has a low frequency inductance of $L_{D}=1.015 \mu \mathrm{H}$ as measured and $L_{B}=0.954 \mu \mathrm{H}$ at the busbar with the clip-leads subtracted. The value is accurate to a few $\%$ at $1.5915 \mathrm{MHz}$, the frequency quoted for most subsequent measurements. The magnet geometry, without end effects, gives a nominal value for the inductance of $L_{M} \approx \mu_{0} h \ell / w=0.706 \mu \mathrm{H}$, clearly below the measured value. The difference can be attributed to end effects and a contribution from the busbar side straps, $L_{S}$. Separating of origins and identification of the strap inductance is required for the interpretation of the "direct" coupling impedance measurements, and will be discussed in the sequel. The resonance at $\sim 28 \mathrm{MHz}$ requires a capacitance of $\sim 33 \mathrm{pF}$ at the busbar terminal and is in part due to the busbar end plates themselves. Subtracting the capacity of the clip leads leaves the plate capacity at $C_{B} \approx 27 \mathrm{pF}$. The resonance of the bare magnet without measuring cable can now be estimated to shift from 28 to $\sim 32 \mathrm{MHz}$, the frequency expected in the TW measurements.

\section{Transformer properties of the kicker magnet}

Following the concept developed in the NS paper, the interaction of the beam with the kicker magnet is best analyzed by means of a model in which the twin wire transmission line and the busbar are considered as a magnetic transformer. The line now represents the primary and the busbar the secondary of the transformer.

The transformer parameters are obtained from the full set of scattering coefficients in which the magnet is coupled to the twin wire line by the mutual inductance. For this measurement, the TW-line is shorted at one end. The magnet is disconnected from the feedthrough and the busbar is accessed with clip leads. The line in the magnet has a characteristic impedance, but in this setup needs not to be matched to the network analyzer impedance, $R_{0}$. An input impedance measurement at the open line terminal, yields the line inductance, $L_{L}$ and the direct measurement at the busbar yields the magnet inductance, $L_{D}$. 
The wire measurements are interpreted with regard to a model represented by the standard electrical engineering equivalent circuit in Fig. 1. This model incorporates the impedance contributions from the coupled kicker inductance, $L_{K}$, the side-strap inductance, $L_{S}=L_{D}-L_{K}$, which is part of the termination, $Z_{g}$, as well as that attributed to the leakage flux, $\left(1-\kappa^{2}\right) L_{L}$. Neglecting the clip lead correction, the mutual inductance, $M$, and the coupling coefficient $\kappa$, are given by

$$
\kappa=\sqrt{\frac{M^{2}}{L_{K} L_{L}}}
$$

and the transformer ratio, $n$ by the geometrical ratio of wire spacing and gap height in kick direction, $n=\frac{M}{L_{K}}=\frac{\Delta}{h}$

The transmission coefficient, $S_{21}$, from the terminals of the Lecher line to the terminals of the bus-bar yields the mutual inductance via simple analytical steps. By considering the transformer model in Fig. 1, and neglecting the presence of any stray capacitance, one can write the low-frequency expression for the scattering coefficient

$$
S_{21}=j \frac{2 \omega M R_{0}}{R_{0}^{2}+j \omega\left(L_{L}+L_{D}\right) R_{0}-\omega^{2}\left(L_{L} L_{D}-M^{2}\right)}
$$

where $R_{0}$ is the instrument impedance, $L_{D}$ the measured bus bar inductance (with the clip lead correction ignored), and $L_{L}$ the line inductance. Note that $L_{D}$ represents the measured value at the busbar, which represents the sum of coupled inductance, $L_{K}$ plus the side strap inductance, $L_{S}$. Finding the value of the side-strap inductance, which during operation actually is part of the magnet termination, $Z_{g}$, becomes necessary to establish the base for further transverse impedance measurements.

Taylor expansion of Eq. (12) to first order in $\omega$ yields the expressions

$$
M \approx \frac{R_{0}}{2 \omega} \operatorname{abs}\left(S_{21}\right) \text { and }\left(L_{L}+L_{D}\right) \approx \frac{R_{0}}{\omega} \operatorname{arc}\left(S_{21}\right)
$$

The low frequency values at $1.5915 \mathrm{MHz}$ are taken from Fig. 9 to be $M=0.131 \mu \mathrm{H}$. The second quantity is in fact a redundant result, $\left(L_{L}+L_{D}\right)=2.494 \mu \mathrm{H}$, and is in reasonable agreement with the directly measured $L_{L}=1.272 \mu \mathrm{H}$ plus $L_{D}=1.015 \mu \mathrm{H}$. The coupled magnet inductance is now found from the mutual inductance to be $L_{K}=M h / \Delta \approx 0.80$ $\mu \mathrm{H}$ (versus the calculated $L_{M}=0.706 \mu \mathrm{H}$ ). Subtracting the relevant values yields the side strap inductance of $L_{S} \approx 0.154 \mu \mathrm{H}$. Seen from the primary beam side, the side strap inductance is part of the external kicker termination and is taken into account for direct coupling impedance measurements via the ratio $L_{K} / L_{D} \approx 0.83$ as corrective factor. As expected, the coupling of TW-line and busbar, when expressed by the coupling coefficient $\kappa \approx 0.13$ is weak. 

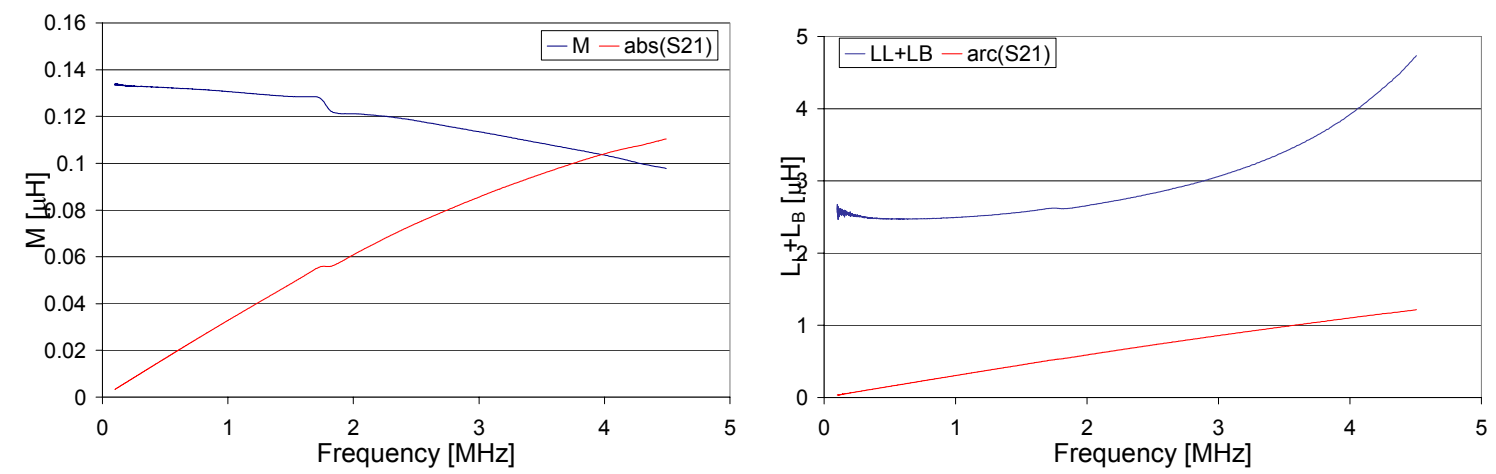

FIG. 9. Mutual inductance from busbar to TW line scattering coefficient

\section{Direct coupling impedance measurement}

The frequency range of interest to SNS covers the frequency range from $\sim 100 \mathrm{MHz}$ down to below $1 \mathrm{MHz}$. Especially at the very-low frequency end, the signals from the wire measurement become very weak and the results appear extremely noisy. This provided the renewed motivation to investigate the wire-less "direct" impedance measurement as a complementary method. Coupling impedance measurements without wires have been reported in the literature [13] and preliminary results for the SNS extraction kicker were obtained with this method $[8,11]$.

In this method, the data is collected directly at the busbar terminal. For the data interpretation, the busbar plates are considered as twin wires with an effective spacing of $h$. The signal level in the direct method is thus increased by $(h / \Delta)^{2}$ over the wire method and it is most advantageous at low frequencies, $<10 \mathrm{MHz}$. The transformer model suggests a complete agreement as to the "coupled" impedance between the direct measurement and the twin-wire by taking the busbar short as reference. Obviously, neither the leakage inductance nor the image currents appear in the direct method. Furthermore, only measurements in the kick direction are possible and only kicker magnets with accessible busbar terminals are accessible. Notwithstanding these limitations, the direct coupling impedance measurement turns out to be one of the better methods below a few MHz. As discussed above, access to the coupled impedance terminals can be restricted by a stray inductance, such as the side strap inductance in the SNS magnet. In this case, a correction to the measured input impedance at the busbar, $Z_{D}$, may be required, leading to

$$
Z_{\perp}=\left(\frac{L_{K}}{L_{D}}\right) \frac{c}{\omega h^{2}} Z_{D} \approx(0.83) \frac{c}{\omega h^{2}} Z_{D}
$$

The low-frequency transverse impedance from the direct (black) and twin-wire measurement (red) of the bare kicker magnet without feedthrough but with $25 \Omega$ termination are compared in Fig. 10. The data from the wire measurement (TW w X300, yellow) becomes noisy below $\sim 3 \mathrm{MHz}$, but is consistent with the direct data (black) after adjustment for the corrective factor of $\sim 0.83$ determined above. 
In this frequency range, the twin-wire line can also be used without transformer or resistive matching to increase the signal strength and to eliminate calibration errors. Transformer-less wire measurements uniquely define the use of the instrument impedance, $R_{0}$ as the nominal impedance, $R_{C}=R_{0}$ rather than the line impedance $Z_{L}$ for the interpretation of the scattering coefficients in Eq. 1. However, there is a systematic error in the scattering coefficient ratio which increases with frequency and is approximated by [4]

$$
j 2 \ell_{L} \frac{\omega}{c} \frac{Z^{D U T}}{Z_{L}} \frac{Z_{L}^{2}-R_{0}^{2}}{\left(2 R_{0}+Z^{D U T}\right)^{2}}
$$

The transformer-less wire method is limited to low frequencies whereas the direct method can be extended to higher frequencies. The agreement with wire measurements confirms the direct method as a valid alternative at least for the resistive transverse impedance measurement. It has the highest signal to noise ratio and in fact can serve here as practical measurement at the lowest frequencies (even without correction).

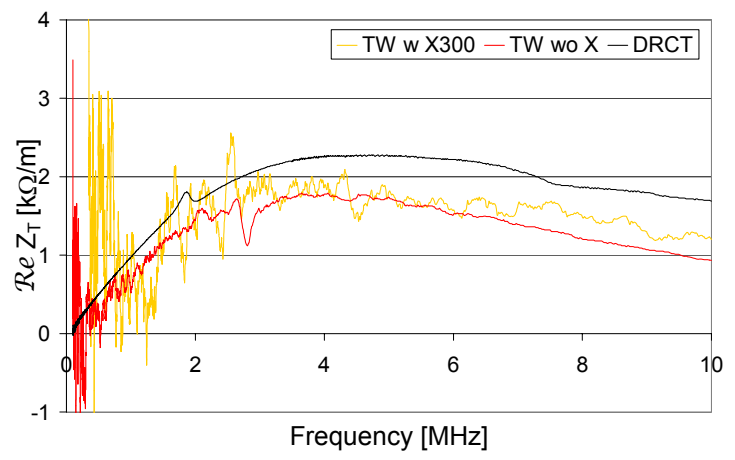

FIG.10. Transverse impedance from wire and direct measurements

\section{The DUT impedance of the prototype kicker magnet}

The DUT impedance of the "bare" SNS kicker magnet, i.e. with the feedthrough and Pulse Forming Network (PFN) disconnected, was measured using the wire as well as the direct method as discussed above. The total impedance seen by the beam is composed of the impedance coupled to the termination plus the impedance due to the image currents. The separate contributions can be identified by making measurements with appropriate configurations on the shorted, open, and terminated magnet respectively.

For the wire measurements, the TW line was assembled with the X200-100MHz or the X200-60MHz and was matched to $R_{C}=250 \Omega$. In order to work with an acceptable signal to noise level, the wire spacing was kept at $\Delta=40.6 \mathrm{~mm}$ to remain in the perturbative region at all frequencies. The validity could be increased at higher frequencies by reducing the wire spacing thereby reducing the coupling coefficient, $\kappa \rightarrow 0$ and the stray inductance which is not seen by the beam. In the present setup with $\Delta=40.6 \mathrm{~mm}$, the measured stray inductance is estimated as $\kappa^{2} L_{L} \approx 0.0215$ $\mu \mathrm{H}$ which is comparable in size to $L_{K}(\Delta / h)^{2}$ seen by the beam. 
Separating the impedance parts involves reference calibration of the TW line, $S_{21}^{R E F}$, versus the tube (vT) or against the shorted busbar (vS). The primary measurement is done versus the tube and yields the total impedance. Measuring the magnet with the busbar shorted, still versus the tube, yields the intrinsic impedance due to the image currents. Subtracting the shorted from the total impedance yields the coupled termination impedance. Alternatively, measuring the magnet versus the shorted busbar too provides the coupled termination impedance and serves as error check.

In the low frequency range of interest to the SNS performance, $\sim 1$ to $10 \mathrm{MHz}$, the image current is reactive and the resistive transverse impedance is primarily due to the coupled termination impedance. It follows that, using the shorted busbar as reference which yields the coupled impedance part alone without the contribution from image currents, entails several practical advantages. The measurement versus short leads to the elimination or at least a reduction of errors due to mechanical changes or drift in the network analyzer. The result for the resistive impedance component, which is of particular interest for the beam stability, remains unchanged by the choice of (loss-less) reference calibration, but the reactive part is modified. Depending on the magnet construction, such as the side straps in the SNS magnet, it may be difficult to achieve a perfect short and small corrections must be applied.

Further measurements were performed using the "direct" method, which can only give the coupled impedance and needs a similar correction as the measurement versus short. In view of the omnipresent errors, correction for the side straps was judged to be unnecessary. The direct results are expected to agree with those from the measurement versus short, and as discussed above, the direct method provides noise free data down to the lowest frequencies.

\section{The shorted Magnet.}

The DUT impedance of the magnet withy shorted busbar is shown in Fig. 11. The magnet was wire measured vs. the tube reference using the X200 transformers, with the transformers identified as to their 100 (X-100, blue) and $60 \mathrm{MHz}$ (X-60, red) frequency range. The wire measurements (red and blue) yield the intrinsic impedance due to the image currents plus a small contribution due to the imperfect short from the shorting strap $(\sim 0.06 \mu \mathrm{H})$. The intrinsic impedance due to the image currents is mostly inductive, but at higher frequencies losses appear in the magnet ferrite (CMD5005 ferrite material with a saturation frequency of $\sim 3 \mathrm{MHz}$ ). The wire measurements with the two transformers (red and blue) agree essentially up to $\sim 20 \mathrm{MHz}$, but differ at higher frequencies and point to the accuracy limit of wire measurements. Overall, the X-100 transformer seems more credible for measurements covering the entire frequency range and will be preferred.

The wire results are shown in Fig. 11 together with the direct data (green), scaled for comparison by the factor $(\Delta / h)^{2}=1 / 37.3$. The direct measurement represents the inductance of the magnet $L_{D}$ with the shorting strap $(\sim 0.06 \mu \mathrm{H})$ in parallel. Since imperfect, the short allows at higher frequencies small busbar currents and some ferrite losses, but the deviations from short are for all practical purposes negligible. 

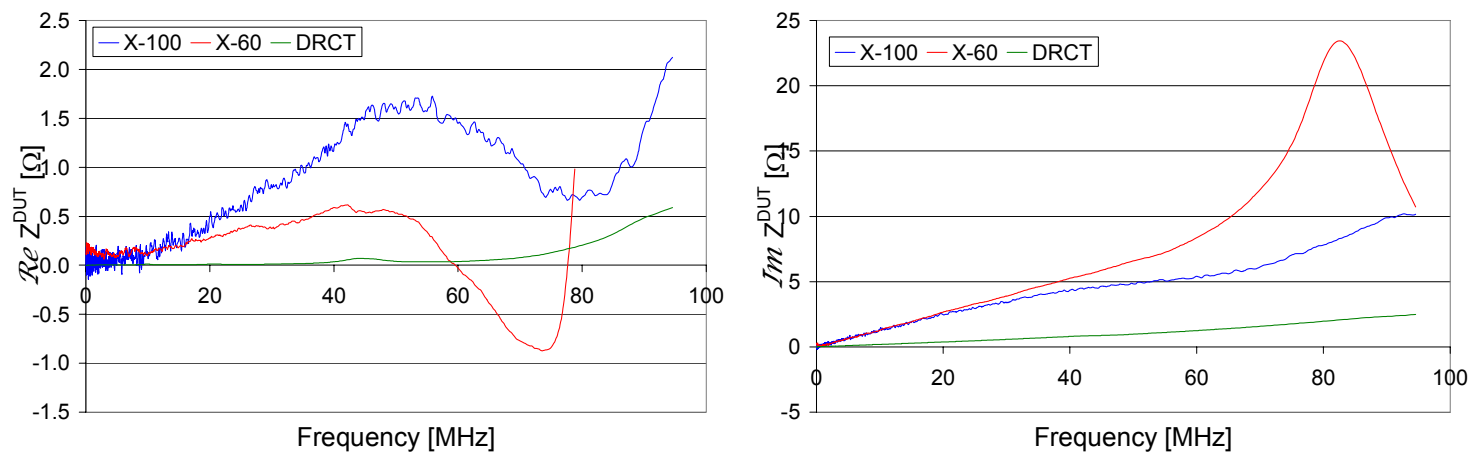

FIG. 11. Measured impedance of the magnet with shorted busbar.

(Note the different scale for real and imaginary component)

\section{The open magnet.}

The magnet impedance with open busbar is shown in Fig. 12. The impedance measured versus tube (vT, blue) is shown together with the measured impedance versus short (vS, red) as well as the direct impedance (DRCT, green). The measurement vT represents the sum of coupled plus intrinsic plus stray impedance. Consistency of the measurements is demonstrated by adding the measured impedance of the open magnet vs $\mathrm{S}$ (red) to that of the shorted magnet vT (SHRTvT) and by comparing the sum (yellow) with the total impedance vT (black). Full agreement is found, even though the three measurements were performed at different times and thus provide an indication of the measuring accuracy.

The direct measurement can only give the coupled impedance and thus is to be compared with the equivalent impedance vS (red). The coupled impedance alone can be represented numerically by the transformer model (MDL, black) with the circuit parameters of $L_{B}=0.954 \mu \mathrm{H}, C_{B}=27 \mathrm{pF}$, and a series $R \approx 12 \Omega$ (or the $\sim 620 \Omega$ parallel resistor of the $Z^{D}$ in Fig. 6). The direct curve (DRCT, green) in Fig. 12 is scaled by the factor $(\Delta / h)^{2}$, but without the $\sim 0.83$ correction; which explains the higher peak in the resistive component. The resonance shift from $\sim 32$ to $\sim 28 \mathrm{MHz}$ is due to the clip lead inductance and capacity. It is seen that the coupled impedance is obtained with sufficient accuracy both from the wire vS and from the direct (even if uncorrected) measurement.

Overall, the various measurement methods give comparable results for the total resistive component of the open magnet. The difference in the reactive part of the total impedance from the wire vT on one hand and the wire vS or direct method on the other is attributable to the image and stray impedances. 

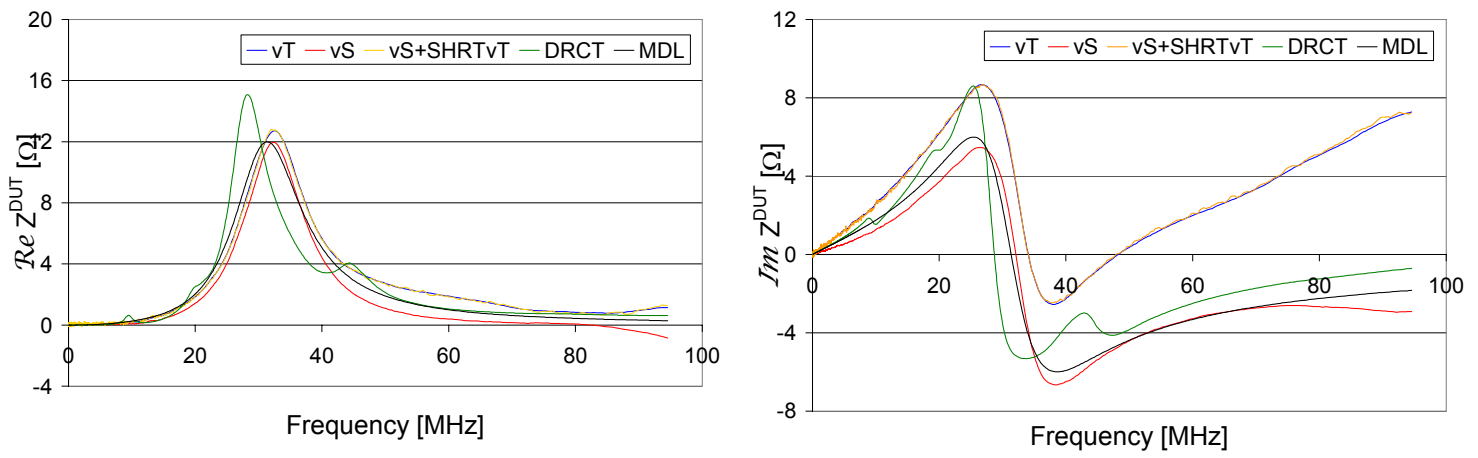

FIG. 12. Measured Impedance of open kicker magnet

\section{The Magnet with $25 \Omega$ termination.}

The measured impedances for the prototype kicker magnet, terminated at the busbar with $25 \Omega$ are shown in Figs. 13 and 14. The nomenclature and colors are here retained from Fig. 12. The numerical level of the impedance is here significantly lowered, which makes the data and their interpretation more sensitive to small errors. The presence of a termination changes the coupled, but not the intrinsic image impedance nor the stray inductance. The results clearly indicated two frequency regions with a qualitatively different behavior. At low frequencies, below $\sim 10 \mathrm{MHz}$, the results are determined by the coupled impedance, dominated by the $25 \Omega$ termination, whereas at higher frequencies the intrinsic, as measured with the shorted magnet, become predominant.
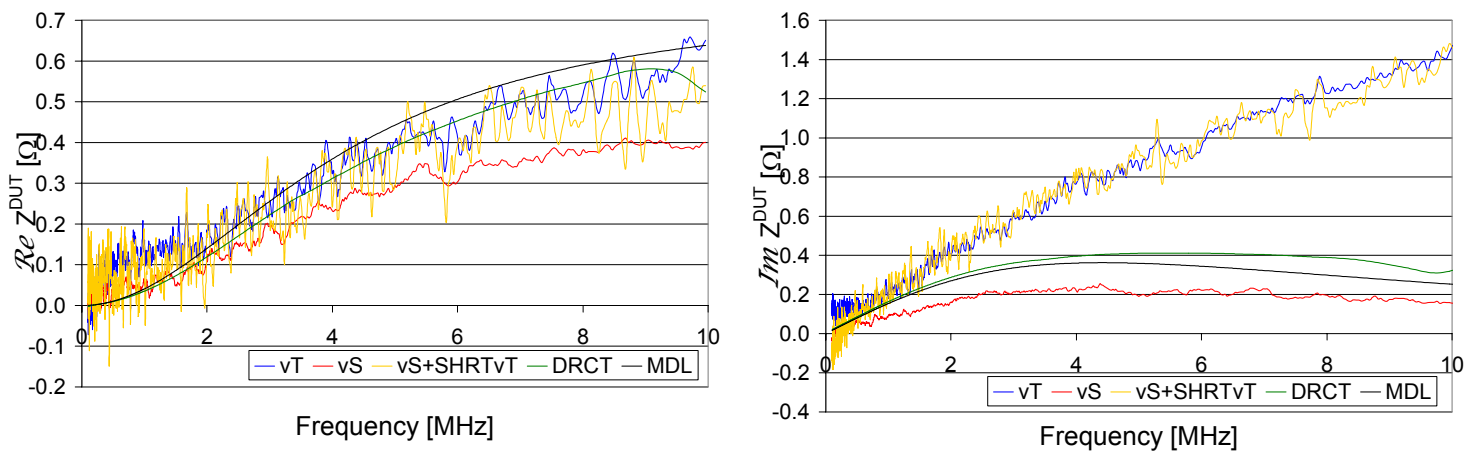

FIG. 13. Measured magnet impedance at low frequencies.
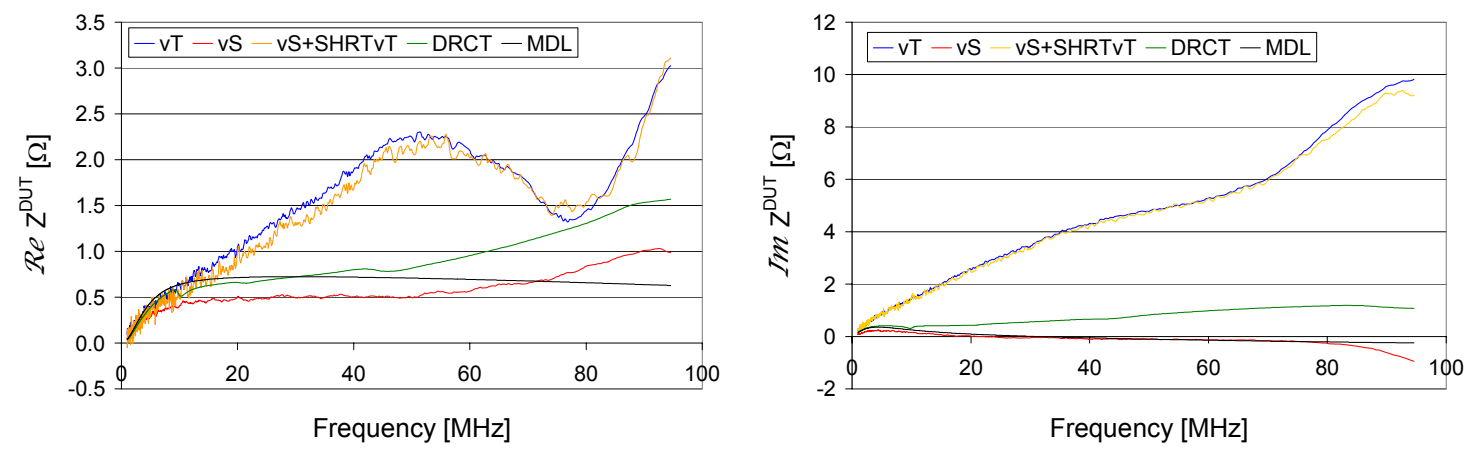

FIG. 14. Measured impedance of magnet with $25 \Omega$ termination 
Interpreting the collective set of magnet measurements confirms wire measurements versus a beam tube calibration as the primary method at all frequencies of interest here. However, the signal to noise ratio decreases at lower frequencies to a degree that the data below a few $\mathrm{MHz}$ becomes marginal. The frequency range of valid data can be extended for the instability driving resistive part of the impedance by making wire measurements versus the shorted busbar as reference. Alternatively, the direct impedance measurement at the busbar provides useable results from $\sim 10 \mathrm{MHz}$ down to $\sim 1 \mathrm{MHz}$, as demonstrated in the previous section with Fig. 10.

\section{The PFN magnet termination}

The kicker magnet is located in a vacuum vessel with feedthrough and is electrically connected to the Pulse Forming Network (PFN) by means of two $\sim 200 \mathrm{~m}$ long $50 \Omega$ cables. The PFN is designed for a $25 \Omega$ output impedance. The cables are in parallel to match to the $25 \Omega$ magnet termination. Wire measurements of the kicker magnet system, that is the magnet with PFN connected, can only be done when the vessel is in air. In air, the magnet busbar terminals as well as the feedthrough terminals at the magnet are accessible. Thus, input (i.e. direct) impedance measurements are also possible on the magnet and on the PFN via feedthrough, separately or fully connected.

Deviations, resistive or reactive, from the nominal $25 \Omega$ PFN impedance lead to frequency dependent impedance oscillations at the busbar terminal and consequently of the kicker coupling impedance. The input impedance seen at the busbar is shown in Fig. 15 for the prototype magnet with $25 \Omega$ (green), as well as for the cables terminated with a "perfect" $25 \Omega$ (blue), or with the first-article PFN (red) as delivered. The results pointed to a $50 \%$ increase of the coupling impedance with the PFN compared to a perfect termination at the busbar.
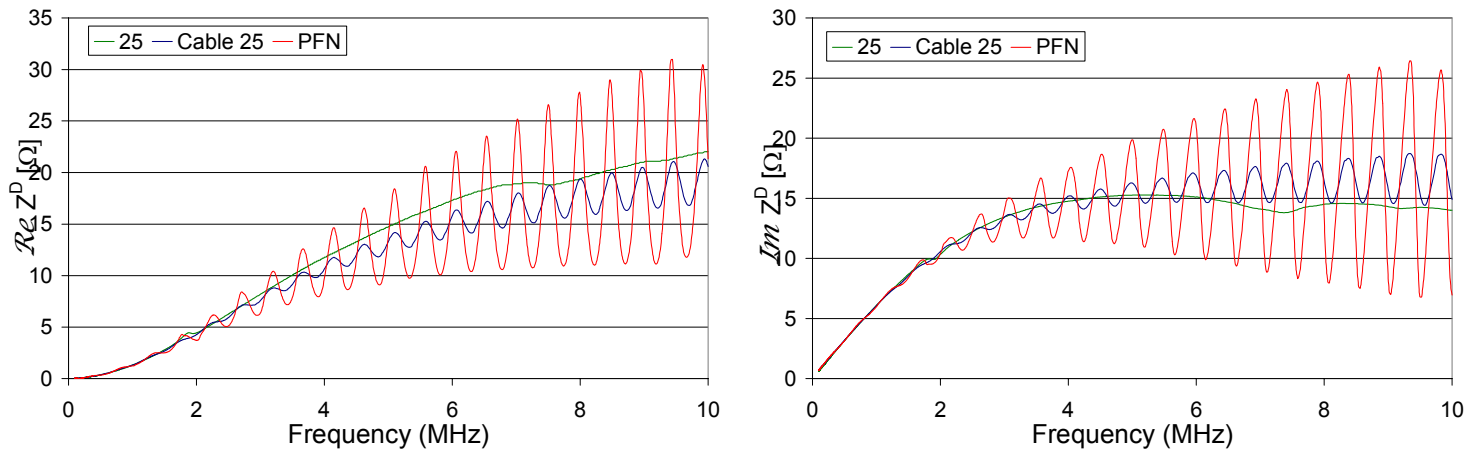

FIG. 15. Impedance at the magnet busbar with $25 \Omega$, cable with $25 \Omega$, or cable with PFN

Impedance measurements of the PFN were made at the terminal to identify the source of the mismatch and to guide in its correction. At first, the presence of a saturating inductor in the PFN was suspected, but shorting it greatly increased the mismatch. Analysis of the initial PFN input impedance (P_type, black), shown in Fig. 16, pointed to its non-zero inductive component as culprit. The inductance of the large PFN load resistor was identified as the inductive source; with the impedance of the free-standing 
resistor (R load, yellow) also shown in Fig. 16. The inductance reduction of the PFN by adding a coaxial screen over the large load resistor was successfully tested (w Scrn, green), and the production units are delivered with load resistor screen.
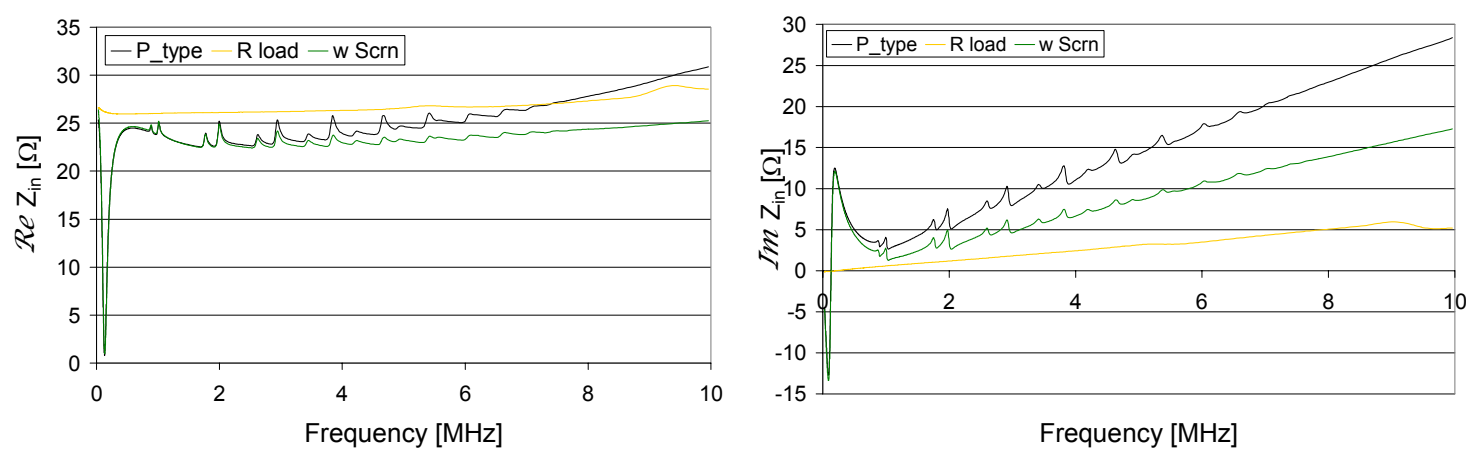

FIG. 16. Input impedance of the PFN.

The improvement from the coaxial screen was demonstrated by measuring the coupling impedance of the "first-article" PFN in its various stages. The dominant instability driving resistive coupling impedance in vicinity of the low frequency peak was obtained from a direct measurement with the scaling of $Z^{D U T}=Z^{D}(\Delta / h)^{2}$ applied. The transverse coupling impedance is shown in Fig. 17. for the PFN without screen (yellow), and as modified with the coaxial screen (blue). The peak value is effectively reduced (by about $30 \%$ ) when compared to the PFN without screen. If necessary, the PFN inductance can be further reduced by adding an RC shunt with a series $R=25 \Omega$ and $C=100 \mathrm{pF}$ across the PFN terminals. The RC shunt alone is beneficial at high frequencies (red), but inferior to the combination of the screen plus a RC shunt (SCRN+RC,green), the solution assumed for the operational condition.

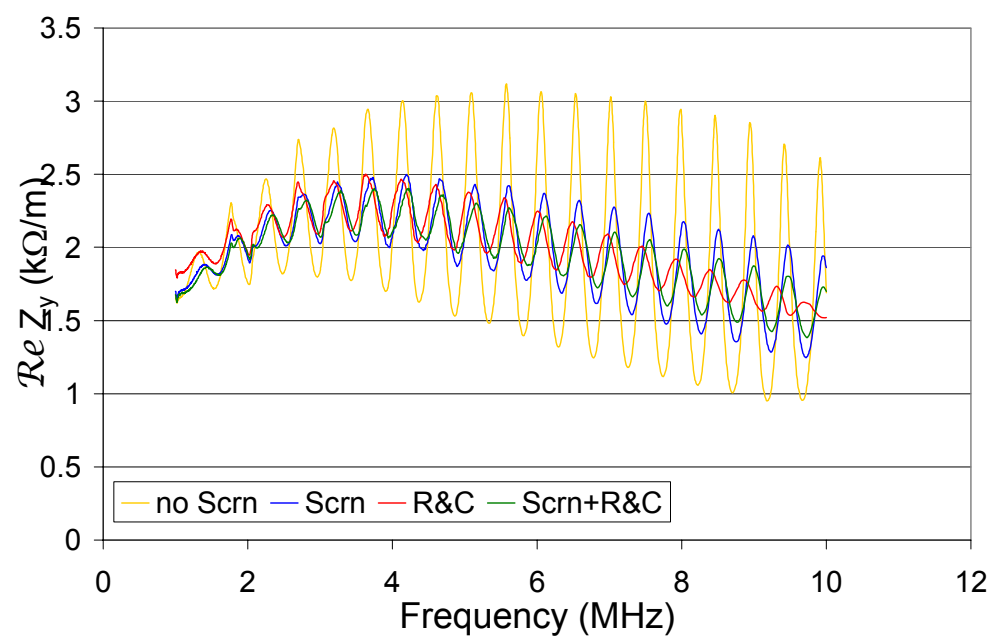

FIG. 17. Transverse impedance with modified production PFN

The $Z^{D U T}$ impedance is significantly reduced by the screen and $\mathrm{RC}$ shunt below $\sim 10$ MHz.. However, the impedance at frequencies beyond $\sim 10 \mathrm{MHz}$ gains less from these modifications as seen in Fig.18. The impedance with screen (blue) is smaller, but not 
significantly below the unscreened (yellow) data. The cables have high-voltage connectors at either end and the feedthrough is a non-matched penetration, so that an irreducible impedance increase over perfectly matched cables must be accepted at higher frequencies.
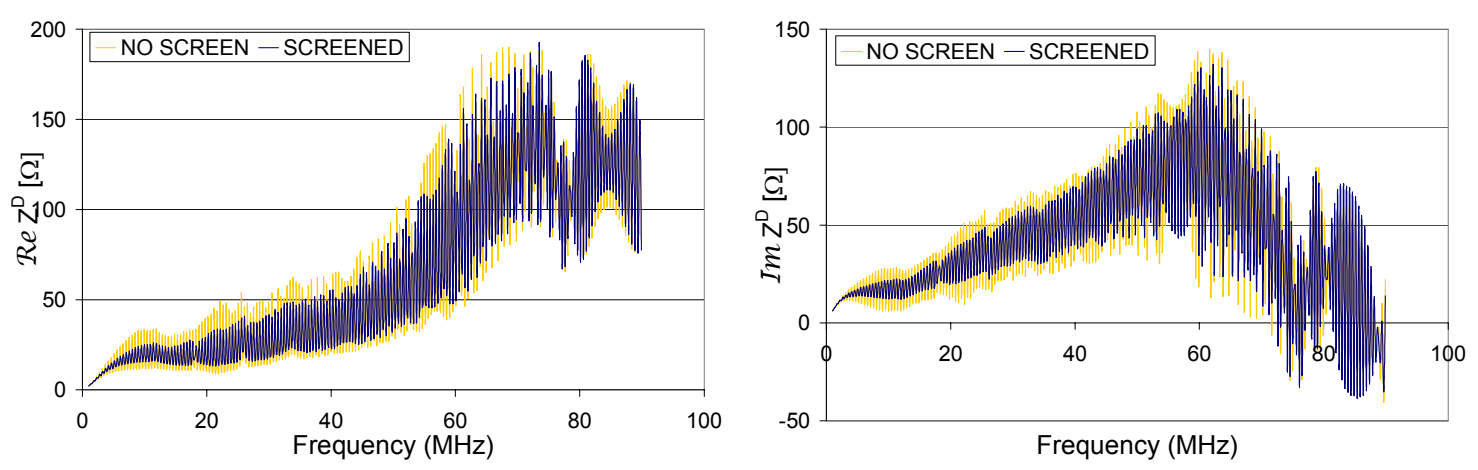

FIG. 18. Impedance seen at the busbar of the magnet with PFN

\section{Transverse coupling impedance of the prototype kicker system}

The transverse impedance is obtained from the "device under test" impedance as discussed in section II using Eq. 2. The transverse impedance differs in horizontal and vertical direction. The vertical impedance, $Z_{y}$ is in kick direction and depends on the kicker magnet termination. The horizontal impedance, $Z_{x}$ has a character similar to the "intrinsic" vertical impedance, and is mostly inductive with a ferrite-generated resistive component. In this section, results for the extraction kicker system consisting of the prototype magnet and the "first-article" PFN after all its modifications are presented and discussed.

\section{Vertical coupling impedance}

The vertical transverse impedance of the kicker system covering the full frequency range of interest, i.e. from $\sim 1$ to $100 \mathrm{MHz}$, was obtained from direct impedance measured at the busbar as well as with the wire method using the appropriate transformers. The measurements were done with the network analyzer setting for a linear frequency sweep, $100 \mathrm{~Hz}$ IF bandwidth, averaged over 3 sweeps, but unless noted otherwise without smoothing. The results are presented in Fig. 19 for the low frequency range and in Fig. 20 for the full frequency range, obtained with the X200-60 and with X200-100 transformer respectively.

The signal to noise ratio below $\sim 10 \mathrm{MHz}$ practically prevents the acquisition of valid data for the resistive component by the wire measurement (TW, yellow), in particular for the resistive peak value at $\sim 4 \mathrm{MHz}$. Here the application of the direct impedance (DRCT, black) is indicated and yields reliable resistive values. The imaginary component can be obtained with the wire measurement (TW, yellow). Alternatively, it is found from the sum of the direct (DRCT, black) plus the intrinsic vS (TW shrt, light blue) values. 

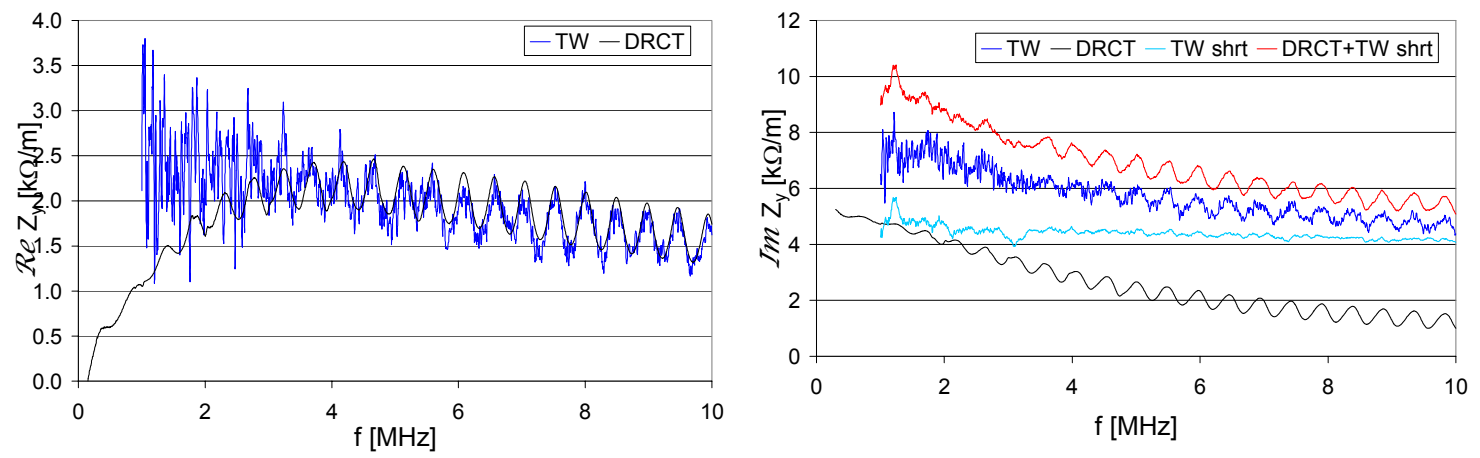

FIG. 19. Vertical impedance of kicker system at low frequencies

The results for the full frequency range in Fig. 20 are obtained with the wire method, both without smoothing $(0 \mathrm{sm}$, blue) and with $0.1 \%$ smoothing $(0.1 \mathrm{sm}$, yellow). Also shown is the image impedance for the shorted busbar (SHRT, red). Adequate overlap of the results in Fig. 20 with those in the low frequency region(Fig. 19) is found.
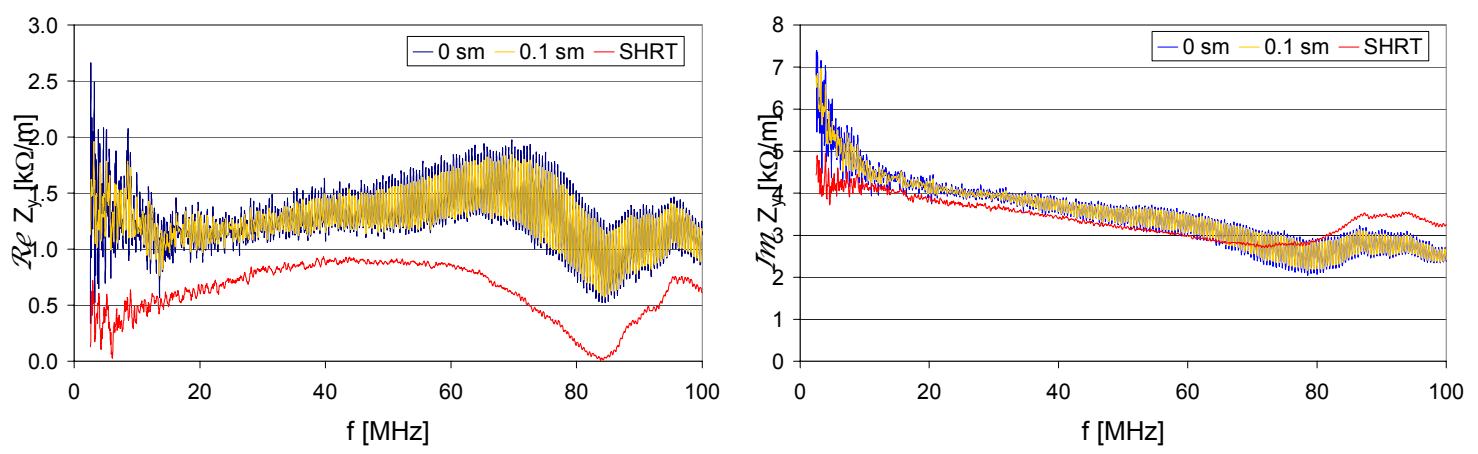

FIG. 20. Vertical impedance of the kicker system

\section{Horizontal impedance.}

The horizontal impedance is independent of the busbar termination and corresponds to the vertical image impedance, and thus it is primarily a reactive value with small ferrite losses. The results for the full frequency range are obtained with the wire method using the X200-100 transformer and are shown in Fig. 21.

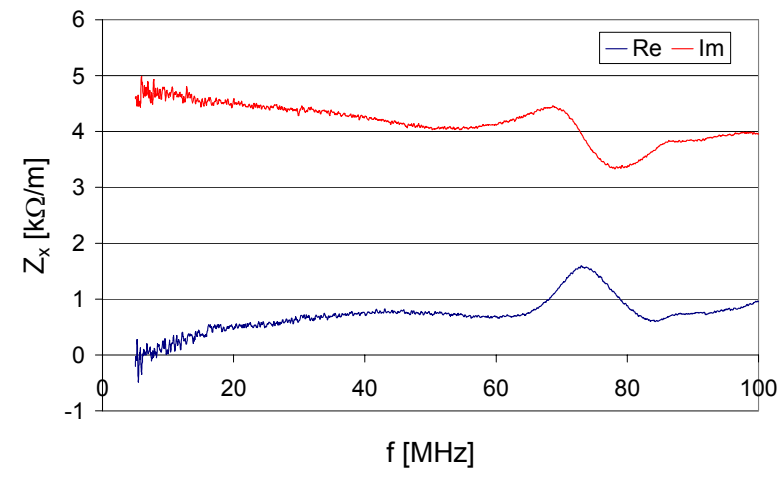

FIG. 21. Horizontal Impedance of the kicker system 


\section{System Impedance with the small-size magnet K1}

The entire SNS extraction kicker system consists of 14 individual magnets. The kicker construction of these magnets is similar, although with different geometrical dimensions. The vertical height of the production magnets ranges from 24.3 to 16.6 $\mathrm{cm}$ measured between the busbar plates, or 24.8 to $17.1 \mathrm{~cm}$ between ferrites. The horizontal width and lengths range from 12.0 to $21.1 \mathrm{~cm}$ and 39 to $51 \mathrm{~cm}$, respectively. The recent availability of the smallest kicker, K1, provided the opportunity for additional measurements which can be used for a review of impedance scaling with geometry. The geometrical dimensions of the $\mathrm{K} 1$ magnet are $h=17.1 \mathrm{~cm}, w=12.0 \mathrm{~cm}$ and $\ell=35$ leading to an inductance of $L \approx \mu_{0} h \ell / w=0.627$ $\mu \mathrm{H}$. The measured input impedance of the open K1 magnet (blue)is shown in Fig. 22 with the inductance at $f=1.5915 \mathrm{MHz}$ as $L=0.871 \mu \mathrm{H}$. Also shown is the input impedance with a 25 ' $\Omega$ termination (red).
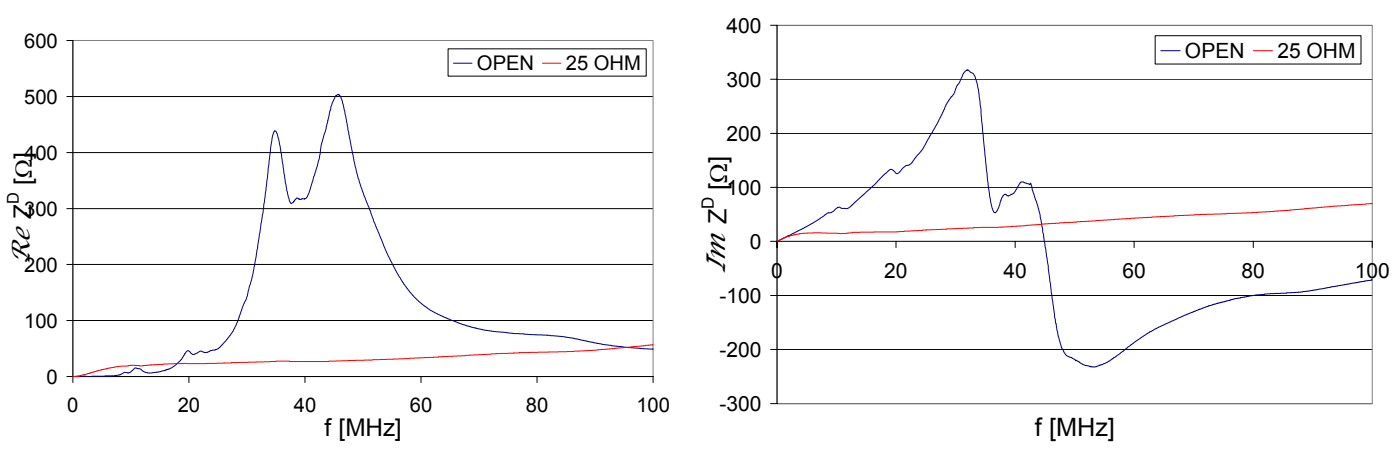

FIG. 22. Input impedance of K1 at busbar.

The low frequency vertical impedance of the K1 magnet with PFN termination was measured both by the direct and the wire method with the low-frequency results shown in Fig. 23. At frequencies at and above the $\sim 4 \mathrm{MHz}$ peak, the two methods agree rather well for the resistive component. One finds at the resistive peak an impedance of $\sim 3.9 \mathrm{k} \Omega / \mathrm{m}$, which is larger than the $\sim 2.4 \mathrm{k} \Omega / \mathrm{m}$ in the prototype magnet by a factor of $\sim 1.63$, but less than the expected vertically scaled $(24.8 / 17.1)^{2} \approx 2.1$ and closer to the horizontally scaled $(15.9 / 12)^{2} \approx 1.76$.

The inductive component is intrinsically less noisy and the best results are obtained with the wire method (blue and yellow). The inductive results for the shorted magnet (light blue) are also shown., whereas the resistive part is negligible. Adding the inductive component from the direct method (black) to the wire value from the shorted magnet leads to a reasonable agreement (red) with the wire method (yellow and blue). 

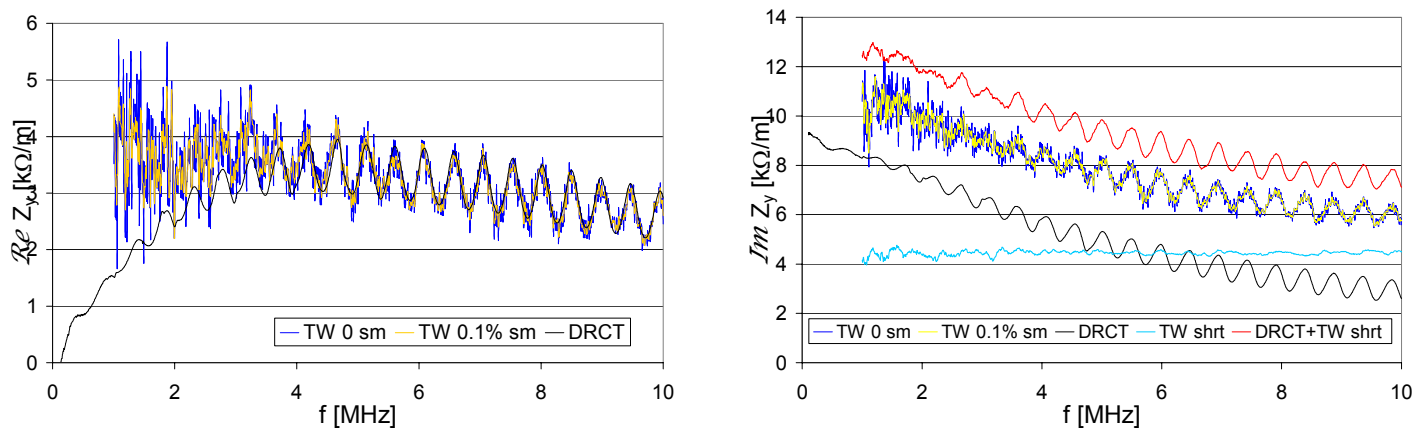

FIG. 23 . Vertical impedance of kicker system with magnet K1

The high frequency measurements were done only with the wire method and without smoothing to retain the oscillations. The results are shown in Fig. 24 for the PFNterminated (blue) as well as for the shorted K1 magnet (red). The peak resistive value of $\sim 4.3 \mathrm{k} \Omega / \mathrm{m}$ is here shifted to a higher frequency, $\sim 90 \mathrm{MHz}$. The real part is clearly dominated by the termination, whereas the inductive part is determined by the image currents. Although the impedance of a single kicker shows the sharp resonances, one can expect the collective total impedance to be somewhat smeared out.
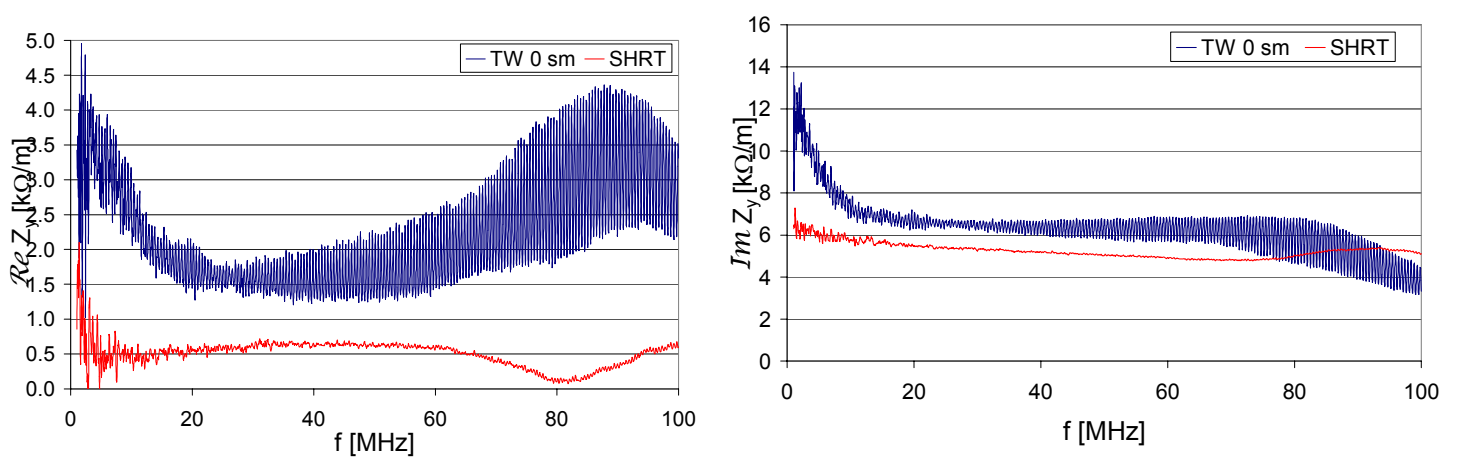

FIG. 24. Vertical impedance kicker system with magnet K1

The horizontal impedance was measured by the wire method and is shown in Fig. 25. The impedance of both, real and imaginary, is independent of the termination and generated by the image currents, and thus primarily inductive.

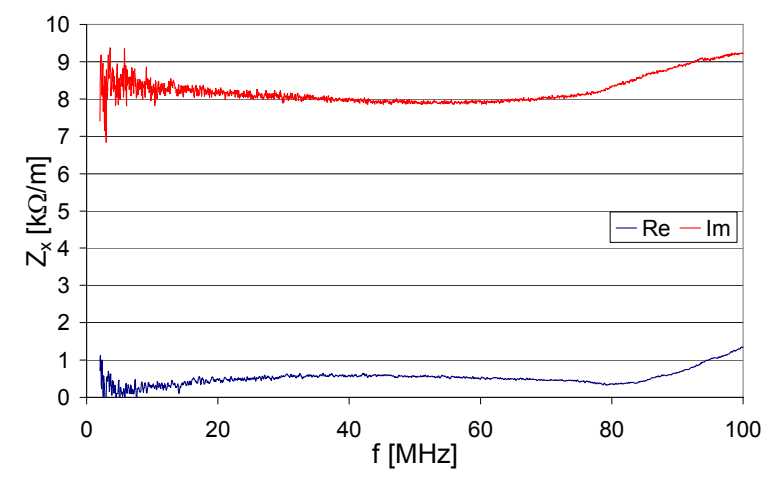

FIG. 25. Horizontal impedance of K1 kicker magnet 


\section{Impedance estimate for the total extraction kicker system}

The availability of the smallest kicker, K1, provided the opportunity for a revised impedance estimate of the total SNS extraction kicker system. The original impedance estimate for the total system was based on the prototype magnet, terminated with a pure resistive $25 \Omega$ termination, and the application of an analytical model.[14] The model includes the magnet geometry and external termination, but its salient feature is the quadratic scaling with the vertical aperture of the kicker magnet. In order to test scaling, the ratio of the vertical system impedance with $\mathrm{K} 1$ divided by that with the prototype is shown in Fig. 26.

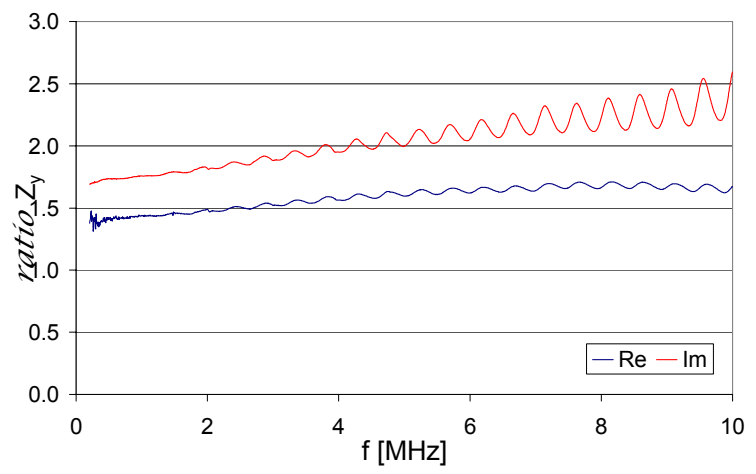

FIG. 26. System impedance ratio of K1 and prototype magnet

Although not perfect, a quadratic scaling can be used for a simplified procedure using only the system impedance of the prototype, $Z_{y}(24.8)$ and of the $\mathrm{K} 1, Z_{y}(16.6)$ magnets as follows

$$
Z_{y} \approx \frac{1}{2}\left[17.6 Z_{y}(24.8)+8.38 Z_{y}(16.6)\right]
$$

leading to the results in Fig. 27. One finds at $\sim 4.5 \mathrm{MHz}$ a resistive impedance peak of $\sim 38 \mathrm{k} \Omega / \mathrm{m}$ which is to be compared with the original estimate of $33.2 \mathrm{k} \Omega / \mathrm{m}$. A secondary peak at the frequency of $\sim 75 \mathrm{MHz}$ has an estimated resistive component of $31 \mathrm{k} \Omega / \mathrm{m}$, which is significantly higher than the original $12 \mathrm{k} \Omega / \mathrm{m}$.
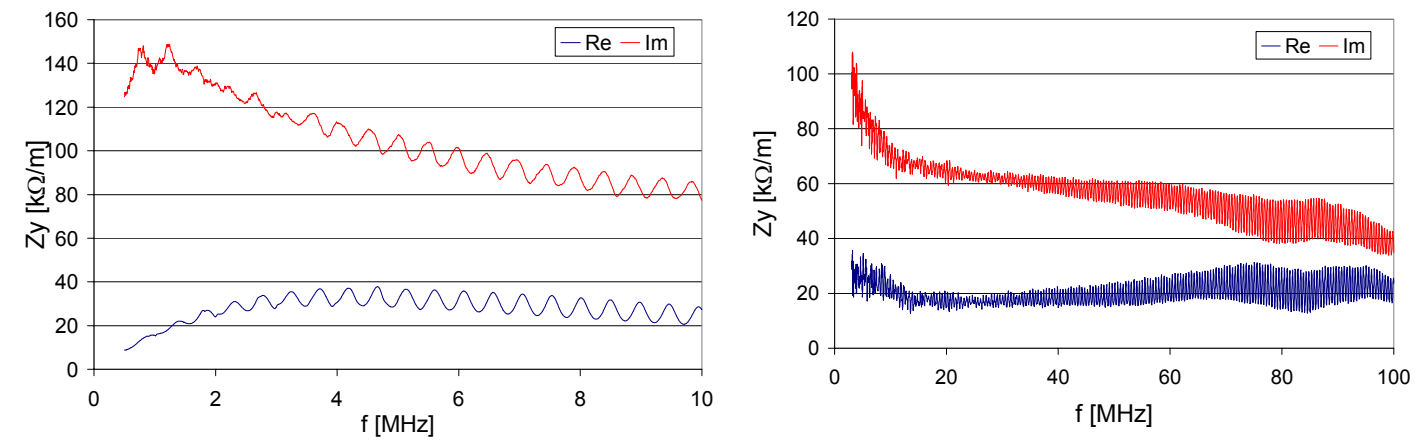

FIG. 27. Estimated coupling impedance of the SNS extraction kicker system 


\section{Acknowledgements}

This paper has greatly benefited from the results of previous work performed together with D. Davino. The measurements presented here were done with the help of D. Warburton. The author would also like to thank C-I. Pai for mechanical engineering help and the members of the Collider-Accelerator Pulsed Power Group, J-L. Mi, W. Zhang and J. Sandberg for general support.

\section{References}

1. F. Caspers, in Handbook of Accelerator Physics and Engineering, edited by A. W. Chao and M. Tigner (World Scientific, Singapore, 1998), p.570.

2. Nassibian and F. Sacherer, Nucl. Instr. Methods 159, 21 (1979).

3. L. S. Walling, D. E. McMurray, D. V. Neuffer and H.A. Thiessen, Nucl. Instr. \& Methods A281, 433 (1989).

4. H. Hahn, BNL Report BNL/SNS Tech. Note, 129, 2003. Phys. Rev. ST Accel. Beams, 6, 012001 (2004)

5. A. Mostacci, F. Caspers, and U. Iriso, Particle Accelerator Conference, Portland, OR, TPPB090, (2003).

6. J. Wei, Proceedings of the 2002 European Particle Conference, Paris, France, 1067 (CERN, Geneva, 2002),

7. J. G. Wang and S.Y. Zhang, BNL Report, BNL/SNS-Technical Note No.79, 2000.

Proc of EPAC 2000, Vienna, Austria, p.972. Nucl. Instr. Meth. Phys. Research A459 (2001), p.381

8. D. Davino and H.Hahn, BNL Reports, BNL/SNS Tech. Note No 88, 2001, BNL/SNS Technical Note No. 102, 2001. Phys. Rev. ST Acce. Beams, 6, 012001 (2003)

9. D. Davino, H,Hahn, and S. Y. Lee, Proc. EPAC 2002, Paris, France ,p. 1467

10. H. Hahn and D. Davino, BNL Report BNL/SNS Tech. Note No 109, 2002. Proc. EPAC 2002, Paris, France, p.1502.

11. H. Hahn, BNL Report BNL/SNS Technical Note 120, 2003.

12. R.E. Collin, Field Theory of Guided Waves, (McGraw-Hill, New York) p.166

13. U. Blell, Proc. of the 1999 PAC, New York, ed. Luccio and W. MacKay (IEEE,Piscataway, NJ, 1999) p. 1727

14. D. Davino, J-L. Mi, and N. Tsoupas, BNL Report BNL/SNS Tech. Note No. 112 (2002) 